\title{
MÉTODOS DE SERIES TEMPORALES EN LOS ESTUDIOS EPIDEMIOLÓGICOS SOBRE CONTAMINACIÓN ATMOSFÉRICA *
}

\author{
Marc Saez (1), Santiago Pérez-Hoyos (2), Aurelio Tobias (3), Carme Saurina (1), M.“ Antònia \\ Barceló (1) y Ferran Ballester (2). \\ (1) Departament d'Economia de la Universitat de Girona, Campus de Montilivi. \\ (2) Institut Valencià d'Estudis en Salut Pública, IVESP, Generalitat Valenciana. \\ (3) Unitat d'Investigació Respiratòria i Ambiental, IMIM, Barcelona. \\ $\left({ }^{*}\right)$ Este trabajo cuenta con una beca del Fondo de Investigaciones Sanitarias (Expediente núm 97/0051).
}

\section{RESUMEN}

Se revisan los métodos de series temporales en los estudios epidemiológicos sobre contaminación atmosférica, ilustrándolo mediante una regresión de Poisson autoregresiva, la cual ha sido utilizada en los proyectos APHEA y EMECAM.

Se relacionan las variaciones en el número diario de muertos mayores de 70 años (todas las causas, CIE-9:001-799) en Barcelona, 1991-1995, con las variaciones en los niveles diarios promedio de contaminación por humos ncgros. Sc utiliza una regresión de Poisson por cuanto la variable aleatoria dependiente sigue presumiblemente tal distribución de probabilidad. Como confusores se consideran variables meteorológicas (promedios diarios de temperatura y de humedad), comportamientos tendenciales, estacionales y efectos de calendario presentes en la mortalidad (todos ellos aproximados de forma determinista) asi como cualquier otra variable que tenga un comportamiento que pueda relacionarse con la variable dependiente (ocurrencia de epidemias de gripe por ejemplo). La relación entre la mortalidad y las variables confusoras se modeliza de forma no lineal y se tienen en cuenta además los previsibles periodos de latencia (utilizando retardos de variables explicativa por ejemplo). Sin embargo, y debido a que el control no es perfecto, se opta por estimar un modelo de Poisson autoregresivo (introduciendo como variables explicativas diversos retardos de la mortalidad) corrigiendo la autocorrelación residual.

La principal ventaja del método de análisis descrito es la de permitir un control de variables confusoras desde un punto determinista, con un software al alcance de todos los grupos que participan en el proyecto. Además, permite que el método se pueda aplicar de una formar protocolizada y estandarizada que facilite la comparación de resultados y permita la realización de un meta-análisis.

Palabras clave: Series temporales. Mortalidad. Contaminación Atmosférica. Regresión de Poisson autoregresiva.

Correspondencia:

Marc Saez

Departament d'Economia

Universitat de Girona, Campus de Montilivi

17071 Girona.

Tel 972-41.87.36, Fax 972-41.80.32,

Correo electrónico: msaez@gnomics.udg.es

\section{ABSTRACT \\ Time Series Methods in the Epidemiological Studies Regarding Air Pollution}

The time series methods in the epidemiological studies on air pollution are reviewed, illustrated by means of an autoregressive Poisson regression which was employed in the APHEA and EMECAM Projects.

A listing is provided of the variations in the daily number of deaths of people over age 70 (all causes, CIE-9:001-799) in Barcelona, 1991-1995, with the average variations in the daily smog pollution levels. A Poisson regression is used insofar as the dependent random variable presumably follows such a probability distribution. As variables possibly leading to confusion, the impact of weather variables (daily temperature and relative humidity averages), seasonal, tendency-related behaviours and day of the ycar on the death rate are taken into account (all estimated on a determinist basis), in addition to any other variable which behaves in a way that it can be related to the dependent variable (i.e. flu epidemics). The relationship between the death rate and the confusing-causing variables is modelled on a non-linear basis, and the foreseeable lag times are also taken into account (i.e. by using explicative variable time lags). However, due to control not being perfect, it has been decided to opt for estimating an autoregressive Poisson model (adding in some different explicative variables time giving rise to a lag in the death rate) offsetting the residual autocorrelation.

The main advantage of the method of analysis described above is that of making it possible to control confusing variables from a determinist standpoint with a software to which all of the groups taking part in this Project had access. This also affords the possibility of using this method in a set, standardized manner, facilitating the comparison of results and making an ohjective point analysis possible.

Key words: Time Series. Death Rate. Air Pollution. Autoregressive Poisson Regression. 


\section{INTRODUCCIÓN}

Una serie temporal es una sucesión ordenada en el tiempo de valores de una variable ${ }^{1}$. El estudio de las series temporales puede consistir en el análisis aislado de una variable o referirse también a la relación entre dos o más de ellas. En la primera aproximación se intenta básicamente entender como evoluciona en el tiempo una variable con el fin de realizar predicciones. En epidemiología, y por lo que se refiere a este trabajo, el interés suele centrarse en el segundo enfoque. En este sentido, se intenta construir un modelo explicativo de la evolución temporal de una variable, con el fin de cuantificar los efectos de factores de riesgo. En los estudios epidemiológicos discutidos aquí, los métodos de series temporales intentan estudiar y cuantificar la asociación entre, al menos, dos series temporales: la serie temporal de la respuesta en términos de un problema de salud, mortalidad por ejemplo, y la serie temporal de exposición, como por ejemplo contaminación.

En primer lugar debemos distinguir los métodos de series temporales de los análisis longitudinales. Los estudios longitudinales se caracterizan porque miden repetidamente a los individuos o sujetos del estudio, a lo largo del tiempo. Contrariamente, los métodos de series temporales suele utilizar observaciones agregadas, lo que en algún momento ha llevado a denominarlos «estudios temporales de datos agregados» ${ }^{2}$. Un ejemplo podría ser el estudio de la asociación entre las variaciones diarias en la contaminación atmosférica y la mortalidad en una ciudad. El número diario de muertos se obtendría del correspondiente registro de mortalidad y los niveles diarios de contaminación atmosférica como promedio de contaminantes registrado en la red de control de la ciudad. Es cierto, sin embargo, que los estudios longitudinales pueden ser analizados de forma agregada (con base diaria, por ejemplo) y que también se han analizado series temporales de individuos ${ }^{3-7}$.
Como segunda diferencia, los períodos de observación en los estudios longitudinales no neccsariamente coinciden en todos los individuos. Los métodos de series temporales, contrariamente, no permiten ni discontinuidades en las observaciones de las variables de interés ni, sobre todo, intervalos de observación de distinto tamaño.

Pero quizás la diferencia más importante radique en que en los métodos de series temporales se supone que existe correlación, o dependencia temporal, entre todas las observaciones de la variable. En un estudio longitudinal sólo existe dependencia dentro de grupos de observaciones, el individuo (del que se disponen de observaciones repetidas) o agrupaciones de análisis, denominadas cluster. Un ejemplo podría ser el del análisis de los factores explicativos de la capacidad pulmonar en un conjunto de individuos. El cluster de análisis lo constituiría el individuo y las observaciones las diferentes mediciones de sus capacidades pulmonares y de posibles variables explicativas. Nótese que los datos se estructuran jerárquicamente, primero el cluster, independiente de otros clusters, y después las observaciones repetidas para cada (y dentro de cada) cluster, dependientes entre $s^{\prime}{ }^{8}$. Estas diferencias exigen de la utilización de métodos estadísticos adecuados y específicos, en algunos aspectos, de cada uno de los distintos diseños.

\section{MÉTODOS DE SERIES TEMPORALES EN EPIDEMIOLOGIIA AMBIENTAL}

Desde el inicio de la utilización de las series temporales en epidemiología, la metodología para analizar la asociación entre el cambio en la exposición promedia y el cambio en la incidencia de la enfermedad ha ido sofisticándose, evolucionando conforme se ha modificado la relación exposición-efecto y/o las variables de interés.

Los primeros estudios epidemiológicos sobre el impacto de la contaminación atmosférica sobre la salud se realizaron como 
consecuencia de los episodios extremos de contaminación. La relación entre la variable dependiente, mortalidad principalmente, y los contaminantes atmosféricos, posibles variables explicativas, se analizaba utilizando básicamente simples representaciones gráficas ${ }^{9}$.

En los países desarrollados tales episodios quedaron circunscritos a los años cincuenta. Desde los sesenta (setenta en nuestro país) los niveles de contaminación atmosférica se redujeron de forma importante. La asociación entre la contaminación atmosférica y algunas variables de salud había sido evidente durante los episodios extremos. Sin embargo, pequeñas variaciones en la calidad del aire podrían tener efectos de menor magnitud en la salud de los individuos. Por tanto se necesitan series temporales más largas a fin de determinar si tales asociaciones son o no estadísticamente si६nificativas. En este sentido diversos trabajos intentaron analizar dichas relaciones utilizando modelos de regresión lineal en los que tanto la variable dependiente como las variables explicativas eran series temporales ${ }^{10-13}$. En general, sin embargo, estos análisis presentan perturbaciones autocorrelacionadas, puesto que, por un lado, relacionan series temporales y, por otro, controlan de forma bastante rudimentaria (y a veces no las controlaron en absoluto) posibles variables confusoras. Al sesgar los errores estándar de los estimadores de los parámetros $y$, en general, reducir la eficiencia de las estimacioncs, la autocorrclación podría poner en serias dudas las inferencias realizadas. Por otra parte, y como es sabido, los modelos de regresión lineal múltiple suponen que la variable dependiente se distribuye normalmente. Quizás no sea ésta una distribución de probabilidad adecuada para variables respuesta de salud.

La metodología del proyecto APHEA ${ }^{14-16}$ supuso un verdadero revulsivo, puesto de manifiesto en los múltiples trabajos que en los últimos años la han utilizado directamente ${ }^{17-21} \mathrm{o}$ se han inspirado en ella ${ }^{22-27}$. En nuestro país el proyecto EMECAM (véanse los trabajos de Pérez-Hoyos et al. y Ballester et al. en este mismo número) pretende evaluar el impacto a corto plazo de la contaminación atmosférica en la salud. Para ello se analizan las series temporales de mortalidad, contaminantes y otras variables aplicando una metodología basada en la utilizada en el estudio APHEA. La metodología propuesta analiza, mediante una regresión de Poisson autorcgresiva, la asociación a corto plazo entre las variaciones temporales en la contaminación atmosférica y en datos agregados de variables de salud, principalmente mortalidad diaria y/o visitas a los servicios de urgencias hospitalarios, prestando especial atención a la estructura de autocorrelación en la variable respuesta, a la estacionalidad y a la tendencia de la misma, y a la influencia de variables meteorológicas.

En el presente trabajo vamos a mostrar las características de la regresión de Poisson autoregresiva para establecer asociaciones entre series de datos temporales en ciencias de la salud.

\section{MÉTODOS}

Como ejemplo para ilustrar el uso del método mostramos en la Figura 1 la evolución temporal del número diario de muertos mayores de 70 años (todas las causas, CIE-9:001-799) en Barcelona, 1991-1995, que intentaremos relacionar con la contaminación.

El número diario de muertos, $Y_{t}$, puede ser considerado una variable aleatoria Poissoniana (figura 2), es decir una variable discreta que toma únicamente valores enteros positivos y cuyo rango, presumiblemente, no es muy amplio (mínimo 12 muertos diarios - máximo 61 muertos diarios). En este caso $Y_{t}$ (denotando por $t$ el día $\left.t=1, \ldots, 1826\right)$ se generará por una distribución de Poisson con parámetro $\mu_{\mathrm{t}}$ (el número diario esperado de muertos mayores de 70 años). 
Figura 1

Número diario de muertos mayores de 70 años. Barcelona, 1991-1995

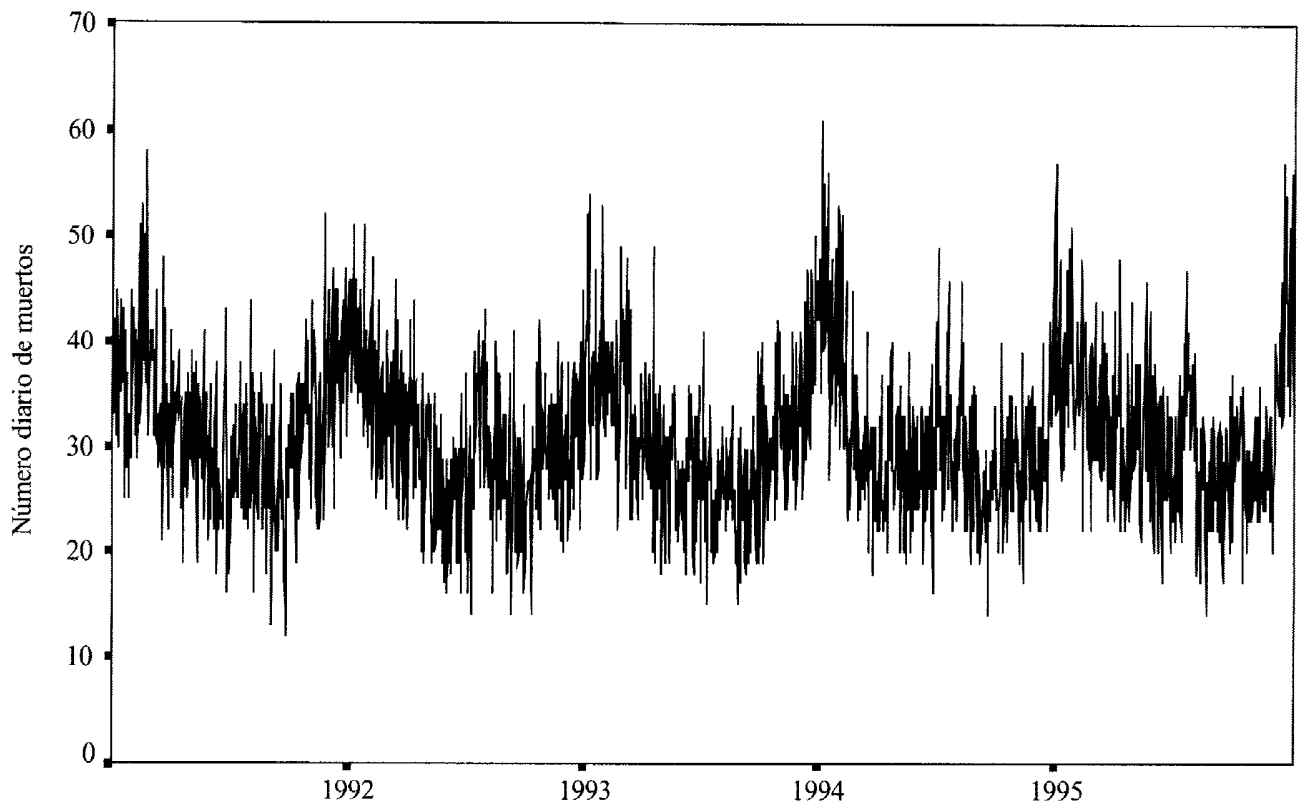

Figura 2

Histograma de frecuencias. Número diario muertos mayores de 70 años

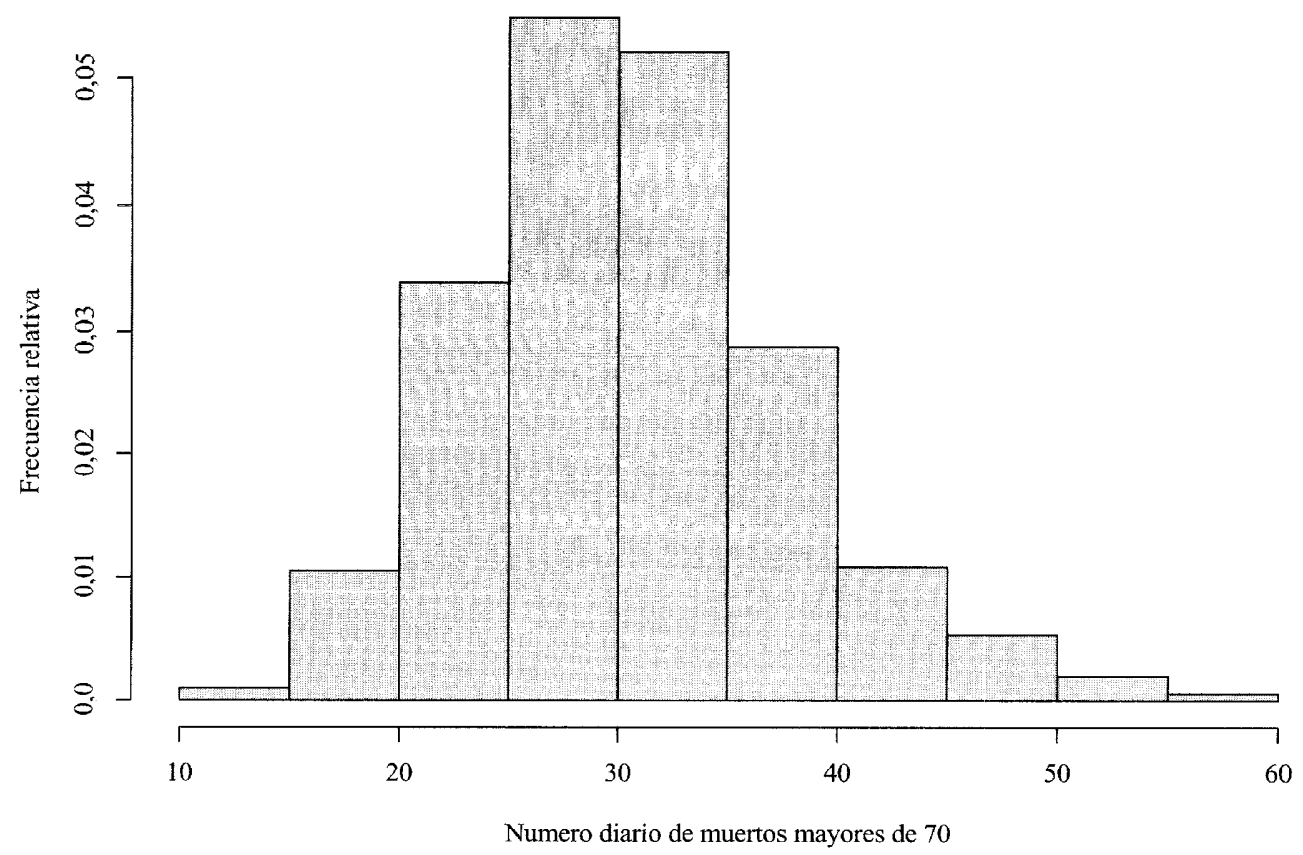


La probabilidad que un día registre exactamente $y_{t}$ muertos mayores de 70 años (siendo $\mathrm{y}_{\mathrm{t}}=0,1,2, \ldots$ ) puede expresarse:

$$
\operatorname{Prob}\left(Y_{t}=y_{t}\right)=\frac{e^{-\mu t} \mu_{t}^{y t}}{y_{t} !}
$$

siendo $E\left(Y_{t}\right)=\mu_{t}$

La variable Poissoniana no es estacionaria en el tiempo, es decir el número esperado de muertos no permanece constante durante todo el período considerado. Así, la serie temporal del número diario de muertos mayores de 70 años presenta una muy ligera tendencia decreciente y un claro comportamiento estacional, con máximos en invierno y mínimos en verano (figura 3 ).

De este modo podemos suponer en nuestro caso que el número diario (medio) de muertos mayores de 70 años, $\mu_{t}$, depende del nivel diario (medio) de contaminantes atmosféricos, por ejemplo humos negros $\left(\mathrm{BLSMK}_{\mathrm{t}}\right)$. Para analizar esta posible relación hay que tener en cuenta algunas posibles variables confusoras. Entre estas se encuentran principalmente variables meteorológicas, como por ejemplo los promedios diarios de temperatura $\left(\right.$ TEMP $_{t}$ ) y de humedad $\left(\mathrm{HUMI}_{\mathrm{t}}\right)$. Así, se puede escribir el siguiente modelo de regresión de Poisson:

$$
\begin{aligned}
\operatorname{Ln}\left(\mu_{\mathrm{t}}\right)=\beta_{0}+ & \beta_{1} \text { BLSMK }_{1}+\beta_{2} \text { TEMP }_{\mathrm{t}}+ \\
& +\beta_{3} \text { HUMI }_{\mathrm{t}}
\end{aligned}
$$

siendo $\beta_{i}(i=0,1,2,3)$ parámetros desconocidos y $t$ denotando el día $t=1, \ldots, 1826$; correspondiendo 1 a 1 de enero de 1991 y 1826 a 31 de diciembre de 1995.

Como instrumento de diagnóstico del ajuste del modelo observamos la evolución temporal de los residuos de Pearson (los residuos más adecuados en este tipo de ajuste). Hay que notar que los residuos presentan una estructura estacional, no oscilando de forma

Figura 3

Número diario de muertos mayores de 70 años y tendencia lineal

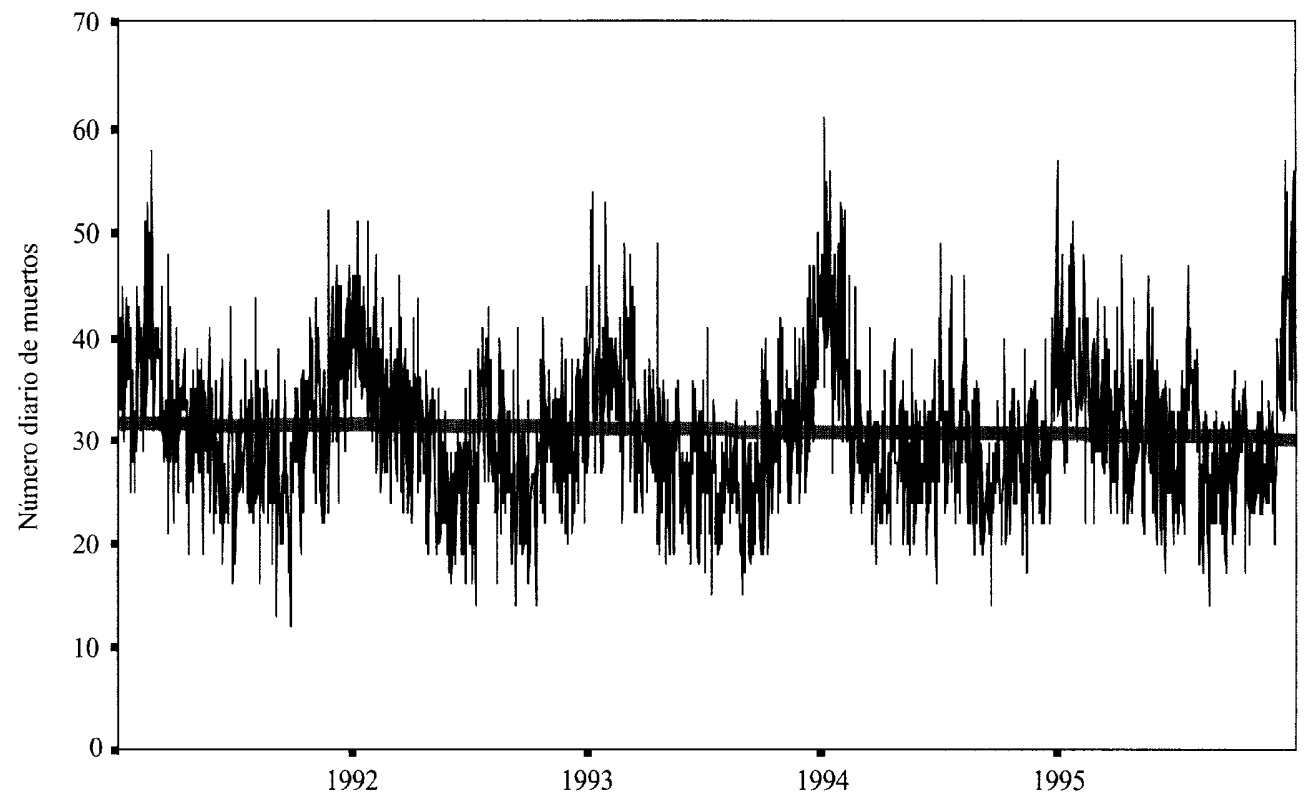


Fvalución temporal de los residuos de Pearson. Regresión de Poisson

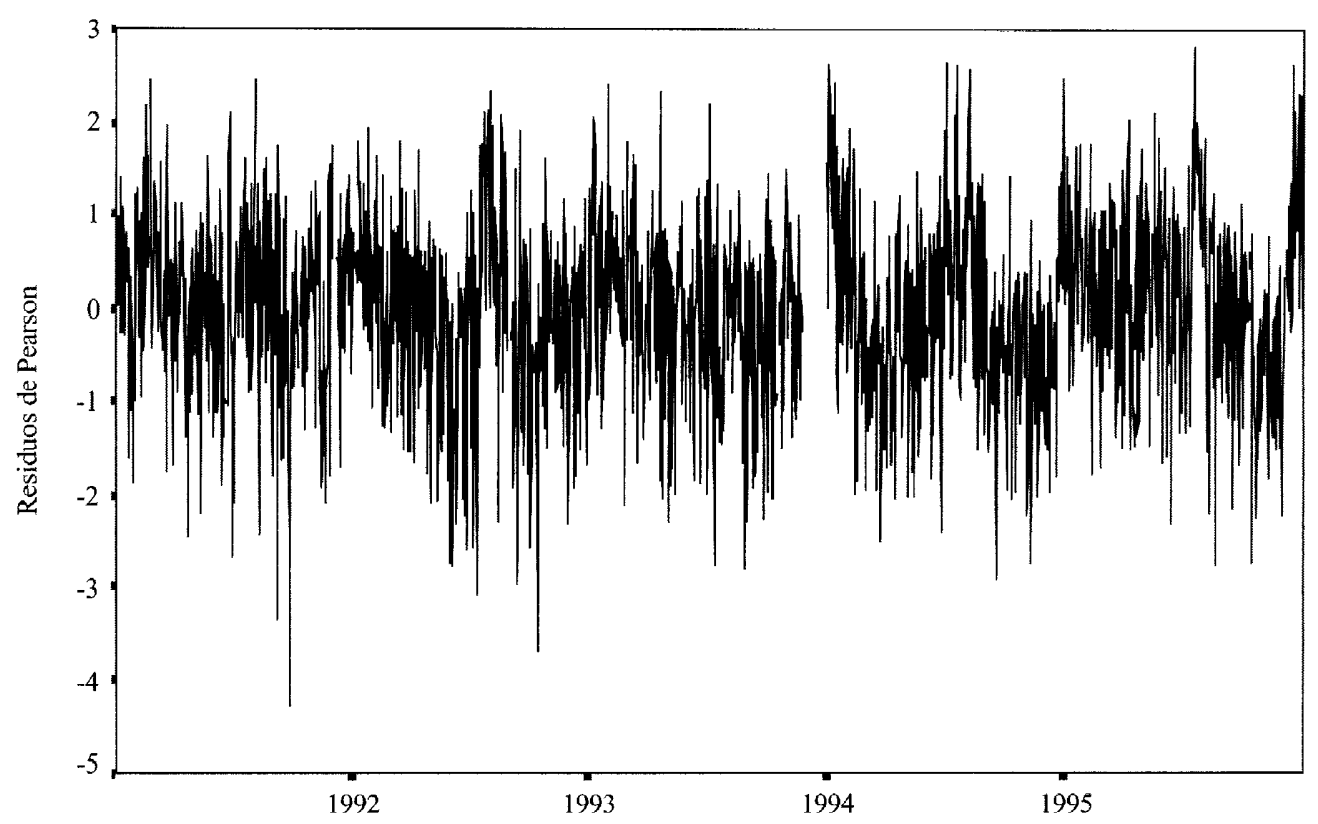

aleatoria en torno al cero con varianza constante. Este hecho podría ser debido a la existencia de errores de especificación. En primer lugar, las relaciones entre la variable dependiente y el contaminante, y entre la primera y las variables meteorológicas podrían no ser lineales, tal y como se ha supuesto hasta ahora. Así, la relación entre la temperatura y la mortalidad puede representarse por medio de la típica forma de $\mathrm{V}$ con el ala izquierda más pronunciada ${ }^{22,28}$. Esta relación se ilustra gráficamente en la Figura 5 mediante el ajuste de una regresión localmente ponderada (lowess) entre el número diario de muertos y la temperatura media.

La relación observada en la figura sugiere que la temperatura (y la humedad) deberian introducirse de forma no lineal en la regresión de Poisson antes expresada.

Por otra parte, no parece muy razonable suponer una relación estática entre los contaminantes y/o las variables meteorológicas y la variable respuesta. Así, por ejemplo, no es lógico suponer que aumentos en la temperatura media por encima de $25^{\circ} \mathrm{C}$ incrementen el número de muertos únicamente $\mathrm{el}$ mismo día, sino que el efecto sobre la mortalidad podía mantenerse algunos días más. Deben tenerse en cuenta los previsibles períodos de latencia (presumiblemente cortos) en la manifestación de los efectos de las variables de interés, introduciendo retardos o promedios de las variables meteorológicas en el modelo de mortalidad.

Además, y tal como pone de manifiesto la función de autocorrelación simple (ACF) de los residuos de Pearson (Figura 6), existe una importante autocorrelación residual en la regresión de Poisson, aún cuando el contaminante (humos negros) y las variables meteorológicas (temperatura y humedad) han sido controlados adecuadamente, es decir la forma funcional y la dinamicidad o latencia de las relaciones es tenida en cuenta. 
Figura 5

Relación entre el número diario de muertos mayores de 70 años y la temperatura media diaria

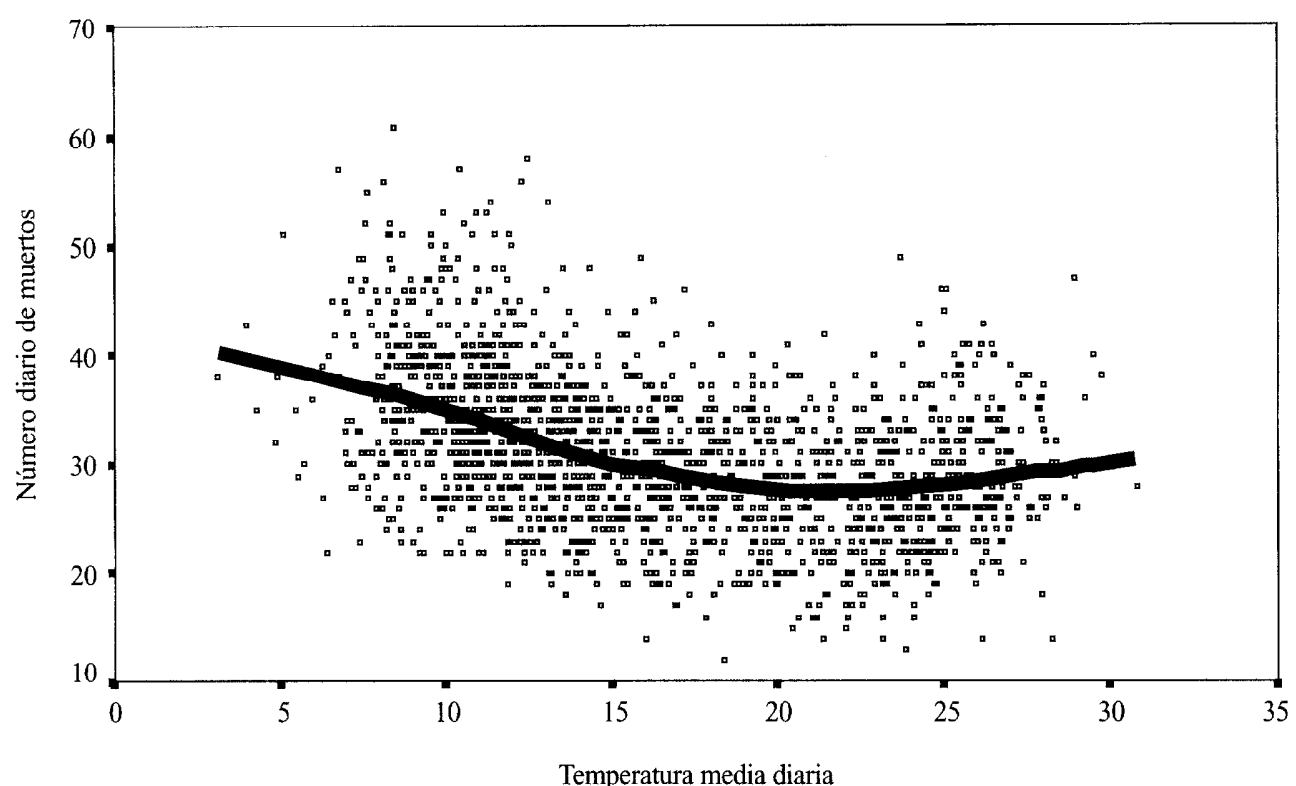

Figura 6

ACF de los residuos de Pearson. Regresión de Poisson

Residual

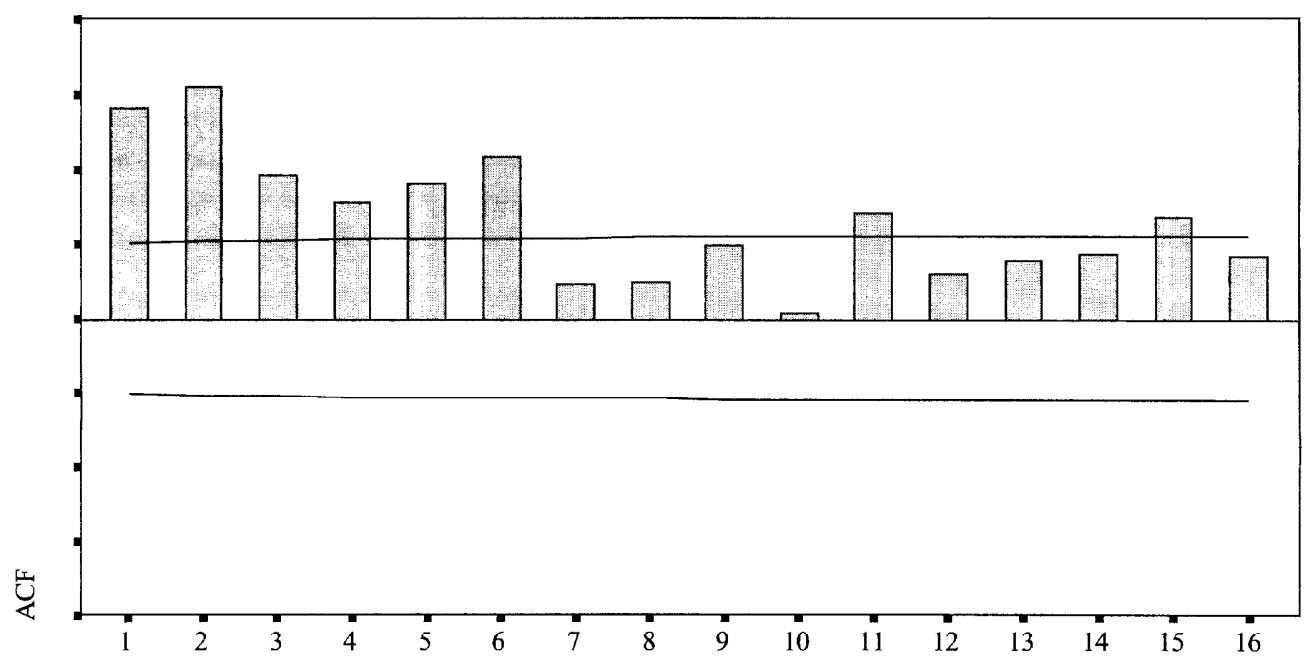

Lag Number

Residuos de una regresión de Poisson: Variable dependiente: Número diario de muertos mayores de 70 años (en logaritmos); Variables explicativas: Humos negros (valor corriente, $t$, y un retardo, $t-1$ ); Temperatura (valor corriente, $t$, uno, $t-1$, tres, $t-3$, y cinco retardos, $t-5$ ); Temperatura al cuadrado (valor corriente, $t$, tres, $t-3$, y cinco retardos, $t-5$ ); Humedad (valor corriente). 
Lo que ocurre es que aún existen importantes confusores que no han sido controlados. En primer lugar ni el contaminante ni las variables meteorológicas explican por completo la ligera tendencia y la estacionalidad de la variable dependiente.

Muchas variables muestran variaciones temporales sistemáticas, conductas sostenidas a largo plazo, es decir tendencias, o conductas rcgulares que se repiten como máximo un año después, denominadas estacionalidad. Por ejemplo, tanto el número de muertos mayores de 70 años en Barcelona como la mortalidad por SIDA en Estados Unidos han venido disminuyendo desde 1991. Por supuesto esto no significa que exista una relación de causalidad entre tales variables. Este mismo tipo de fenómeno puede darse al analizar la relación entre las variables meteorológicas y el contaminante con la mortalidad. Dado que cualquier par de variables que presenten tendencia estarán correlacionadas, la tendencia debe ser eliminada de la posible relación causal.

Además, la mayoría de series temporales presenta un comportamiento estacional. Las variaciones estacionales en el número de muertos mayor de 70 años podrían estar causadas además de por factores meteorológicos, por otros factores que también tengan un comportamiento estacional. Estas conductas deberían ser eliminadas de las posibles relaciones causales. Finalmente, los efectos denominados «de calendario», como los del día de la semana o los efectos de las fiestas, podrían también producir algún sesgo en la estimación de relaciones causales entre series temporales, por lo que deben ser controlados.

Existen dos formas de controlar las variaciones temporales sistemáticas: la aproximación estocástica y la aproximación determinista. En numerosos estudios de relación entre la mortalidad y la contaminación, como el proyecto APHEA o el proyecto EMECAM que se presenta posteriormente en esta misma revista, se opta por utilizar una aproximación determinista de las variaciones temporales. En series temporales con un número pequeño de años, como suele ser el caso de los estudios que estamos comentando, es previsible que no se observen grandes variaciones en la evolución de la componente de tendencia. Por ello se suele optar por construir una función lineal o polinómica del tiempo transcurrido desde el origen del estudio, aproximación de tipo determinista. Por otra parte las variaciones estacionales, es decir el hecho de que el número de muertos sea superior en invierno que en verano, sigue un patrón parecido en los períodos de estudio, observando además que la transición entre la estación cálida y la fría es más suave que entre la estación fría y la cálida. Esta conducta también puede ser controlada de modo determinista, bien incluyendo variables ficticias que contemplen los meses del año, las estaciones climatológicas, etc o bien incluyendo términos sinusoidales del tipo $\operatorname{sen}(\mathrm{k} 2 \pi \mathrm{t} / 365)$ y $\cos (\mathrm{k} 2 \pi \mathrm{t} /$ 365) siendo $\mathrm{k}$ un valor de 1 al 6 y $\mathrm{t}=1,2, \ldots$ el número de días transcurridos desde el inicio del estudio. Este tipo de términos permite recoger desde ciclos anuales $(\mathrm{k}=1)$ hasta ciclos bimestrales $(\mathrm{k}=6)$.

Además de los factores estacionales propiamente dichos, como se ha comentado, los efectos de calendario pueden ser controlados mediante variables ficticias deterministas. De este modo se pueden controlar los días de la semana, tomando el lunes como referencia, los días de fiesta, etc. Finalmente, deberían controlarse otros posibles confusores tales como la ocurrencia de epidemias de gripe o algún suceso inusual que tenga un comportamiento que pueda relacionarse con la variable dependiente.

Tras controlar las variaciones temporales sistemáticas, a pesar de observar una importante reducción, la figura 7 muestra como existe aún autocorrelación residual en la regresión de Poisson finalmente estimada. Ello ocurre porque no se han podido controlar todos los posibles confusores ni se ha po- 
Figura 7

ACF de los residuos de Pearson. Regresión de Poisson

Residual

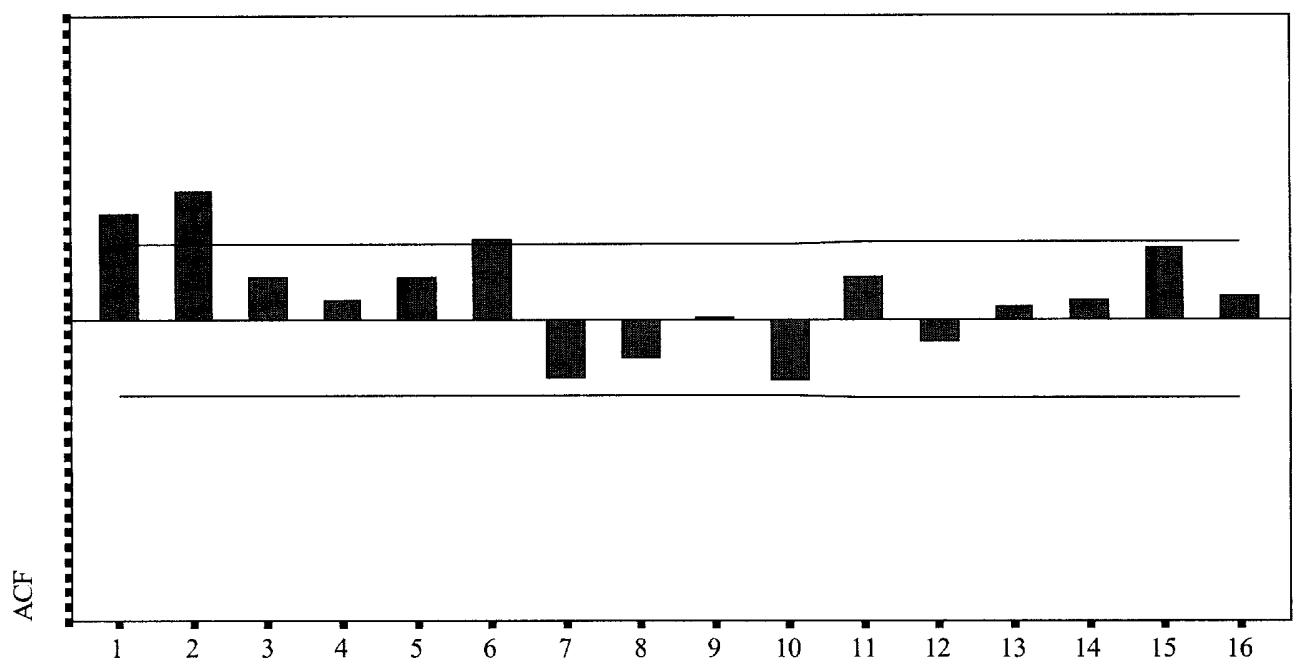

Lag Number

Residuos de una regresión de Poisson: Variable dependiente: Número diario de muertos mayores de 70 años (en logaritmos); Variables explicativas: Humos negros (valor corriente, $\mathrm{t}$, y un retardo, $\mathrm{t}-1$ ); Temperatura (valor corriente, $t$, uno, $\mathrm{t}-1$, tres, $\mathrm{t}-3, \mathrm{y}$ cinco retardos, $\mathrm{t}-5$ ); Temperatura al cuadrado (valor corriente, $t$, tres, $t-3$. y cinco retardos, $t-5$ ); Humedad (valor corriente); Tendencia lineal ( $t=1, \ldots, 1826)$; Variables ficticias para los años (a1992=1 1992, 0 otro caso; a1993, a 1994 y a 1995); Términos sinusoidales $(\operatorname{sen}(\mathrm{k} 2 \pi / 365)$ y $\cos (\mathrm{k} 2 \pi / 365)$, $\mathrm{k}=1,2,4,5$ y 6$)$; Efectos calendario (Martes: 1 Martes, 0 otro caso; Miércoles, Jueves, Viernes, Sabado. Domingo); riesta ( 1 festivos no Domingo, 0 otro caso); B92 (1 desde el 15 julio 15 hasta el agosto de 1992,0 otro caso); Número diario de casos de gripe (valor corriente, $t$, uno. $t-1$, tres, $t-3, y$ cinco, $t-5$, retardos).

dido representar exactamente la relación entre la variable dependiente y cada una de las explicativas. Cabe recordar que la presencia de autocorrelación si bien no sesga la estimación de los parámetros, proporciona errores estándar erróneos y no eficientes, lo que invalidaría las inferencias que hubieran podido realizarse.

Por este motivo se opta por corregir la autocorrelación residual estimando un modelo de Poisson autoregresivo. De entre todos los modelos autoregresivos escogemos el propuesto por Besag ${ }^{29}$, dado que es el único que puede ser estimado recurriendo a métodos y a software estándar ${ }^{30}$. El modelo se construye introduciendo como variables ex- plicativas retardos de la variable dependiente. En el caso que se presenta, y tras observar la función de autocorrelación simple de los residuos (figura 7), se consideró que deberían introducirse los retardos 1,2 y 6 (aunque este último parece sólo marginalmente significativo).

Así el modelo final ajustado queda como:

$$
\begin{aligned}
\operatorname{Ln}\left(\mu_{\mathrm{t}}\right) & =\beta_{0}+\sum \beta_{\mathrm{i}} \mathrm{x}_{\mathrm{it}}+\gamma_{1} \mathrm{Y}_{\mathrm{t}-1}+ \\
& +\gamma_{2} \mathrm{Y}_{\mathrm{t}-2}+\gamma_{3} \mathrm{Y}_{\mathrm{t}-6}
\end{aligned}
$$

en el que $Y_{t}$ denota el número diario de muertos mayores de 70 años en el tiempo $t y$ $\mathrm{x}_{\mathrm{it}}$ las variables explicativas vistas anteriormente. 


\section{DISCUSIÓN}

La principal desventaja de los métodos de series temporales, al igual que otros estudios ecológicos basados en datos agregados, radica en la posibilidad de incurrir en la conocida falacia ecológica. Sin embargo, la posibilidad de confusión es menor que en los estudios geográficos (agregados). De hecho, gran parte de los confusores potenciales pueden suponerse más o menos constantes en el tiempo y, en todo caso, muy poco correlacionados con la exposición. Pero además, la gran ventaja de los métodos de series temporales respecto a otros diseños ecológicos, radica en la posibilidad de controlar la confusión tiempo-dependiente, incluso la altamente correlacionada con la exposición, introduciendo tendencias u otras variaciones sistemáticas en las relaciones causales.

Como ventaja respecto a los análisis de base individual, los métodos de series temporales permiten considerar las variaciones temporales en la exposición. Estas variaciones no siempre son observables en estudios de índole individual. Por otra parte, y a diferencia de los análisis individuales, los métodos de serics temporales suclen utilizar gran cantidad de datos, lo que permite aumentar la potencia estadística $y$, por tanto, detectar débiles asociaciones entre respuesta y exposición. Los métodos de series temporales, finalmente, suelen ser bastante baratos al utilizar medidas de exposición y de respuesta comunes a toda la población.

El método de análisis descrito, y que posteriormente es presentado como el utilizado por el proyecto EMECAM, tiene entre sus principales ventajas, el permitir un control de variables confusoras desde un punto determinista con un software al alcance de todos los grupos que participan en el proyecto. Además permite que el método se pueda aplicar de una formar protocolizada y estandarizada que facilite la comparación de resultados y permita la realización de un meta-análisis.
Alternativamente, la confusión se podría haber controlado utilizando una aproximación estocástica, aunque las variaciones sistemáticas en las variables respuesta no inducen a pensar en un comportamiento de este tipo, $y$ un enfoque determinista puede resultar suficiente. Por otra parte, el modelo Poisson autoregresivo únicamente es estacionario (conducta deseable) si la suma de los estimadores de los parámetros de los retardos de la variable dependiente tiene signo negativo ${ }^{30}$. Este hecho se produce en la mayoría de las ocasiones y la comprobación debe de efectuarse al final del proceso de análisis. En el caso de que finalmente no se confirmara la estacionalidad del proceso se deberían utilizar otro tipo de modelos bien de tipo Markovianos estacionarios sin imponer restricción o bien modelos mixtos de Poisson de efectos aleatorios, que pudieran recoger las variables confusoras no incluidas y que permitieran explicar la heterogeneidad aparente de los modelos. Sin embargo, el ajuste de estos modelos requiere la utilización de programas estadísticos complejos y específicos, con lo cual serán únicamente considerados en el caso de que los modelos de Poisson autoregresivos mencionados en este trabajo nos hagan sospechar la existencia de graves problemas de heterogeneidad de los modelos.

\section{BIBLIOGRAFÍA}

1. Murillo C. Métodos Estadísticos de Series Temporales. Barcelona: SG Editores, 1994.

2. Comission of the European Communities. Study designs. COST 613/2 Air Pollution Epidemiology Reports Series. Report 4. Directorate-General XII for Science, Research and Development. EUR 15095 EN. Luxembourg; 1993.

3. Crabtre BF, Ray SC, Schmidt PM, O'Connor PJ y Schmidt DD. The individual over time: time series applications in health care research. J Clin Epidemiol 1990, 43(3):241-260.

4. Busso T, Liang PJ y Robbins PA. Breath-tobreath relationships between respiratory cycle variables in humans at fixed end-tidal $\mathrm{PCO} 2$ and PO2. J Applied Physiol 1996, 81(5):2287-2296. 
5. Imhoff $M$ y Bauer $M$. Time series analysis in critical care monitoring. New Horizons 1996, 4(4):519-531.

6. Middelkoop HA, van Dam EM, Smilde-van den Doel DA y Van Dijk G. 45-hour continous quintuple-site actimetry: relations between trunk and limb movements and effects of circadian sleep-wake rhythmicity. Psychophysiol 1997, 34(2):199-203.

7. Watanabe I, Imai S, Ikeda M y Ishida A. Time series analysis of the course of Meniere's disease. Act Otolaryngol Suppl 1997, 528:97-102.

8. Cnaan A, Laird NM y Slasor P. Using the General Linear Model to analyse unbalanced repeated measures and longitudinal data. Stat Med 1997, 16:2349-2380.

9. Logan WPD. Mortality in the London fog incident, 1952. Lancet 1953, 264:336-338.

10. Martin AE y Brandley WH. Mortality, fog and atmospheric pollution: An investigation during the winter of 1958-59. Monthly Bulletin of the Ministry of Health 1960, 19:56-71.

11. Hodgson TA. Short-term effects of air pollution on mortality in New York city. Env Sci Tech 1990, 4:589.

12. Schimmel la y Greenburg L. A study of the relation of pollution to mortality, New York city 1963-1968. J APCA 1972, 22:607-616.

13. Buechley RW. $\mathrm{SO}_{2}$ levels, 1967-72 and perturbations in mortality. Report to the National Institutes of Health (NIEHS). Contract NO1-ES-5-2101, 1975.

14. Katsouyanni K, Zmirou D, Spix C, Sunyer J, Schouten JP, Pönkä A, Anderson HR, Le Moullec Y, Woityniak B, Vigotti MA et al. Short-term effects of air pollution on health: a European approach using epidemiological time-series data. The APHEA project: background, objectives, design. Europ Respir J 1995, 8(6):1030-1038.

15. Schwartz J, Spix C, Touloumi G, Bachárová L, Barumamdzadeh T, le Tertre A, Piekarski T, Ponce de León A, Pönkä A, Rossi G, Saez M y Schouten J.P. Methodological issues in studies of air pollution and daily counts of deaths or hospital admissions. J Epidemiol Community Health 1996, 50(Suppl. 1):S3-S11.

16. J Epidemiol Community Health, 1996, Vol 50, Supplement 1.

17. Vigotti MA, Rossi G, Zanobetti A, Benvenuti A, Repetto F, Bisanti L, Gianelle V, Lavecchia C y Azzolini L. Inquinamento atmosferico e mortalita giornaliera nei residenti a Milano, 1980-89. Risultati preliminari. Epidemiol Prev 1995, 19:85-89.
18. Rossi G, Zanobetti A y Marchi M. Analisi di serie temporali in epidemiologia ambientale: effetti a breve termine dell'inquinamento atmosferico sulla mortalita e morbosita. Epidemiol Prev 1995, 19:90-98.

19. Pönkä A. Assessment of the impact of ambient air pollutants on health in Helsinki, Finland. World Health Stat Q 1995, 48(2):126-131.

20. Ballester F, Corella D, Pérez-Hoyos S y Hervás A. Air pollution and mortality in Valencia, Spain: a study using the APHEA methodology. J Epidemiol Community Health 1996, 50(5):527-533.

21. Poloniecki JD, Atkinson RW, de Leon AP y Anderson HR. Daily time series for cardiovascular hospital admissions and previous day's air pollution in London, UK. Occup Environ Med 1997, 54(8):535-540.

22. Saez M, Sunyer J, Castellsagué J, Murillo C y Antó JM. Relationship between weather temperature and mortality: a time series analysis approach in Barcelona. Int J Epidemiol 1995, 24(3):576-582.

23. Loomis DP, Borja-Aburto VH, Bangdiwala SI y Shy CM. Ozone exposure and daily mortality in Mexico City: a time-series analysis. Res Rep Health Eff Inst 1996, 75:1-45.

24. Delfino RT, Murphy-Moulton AM, Burnett RT, Brook JR y Becklake MR. Effects of air pollution on emergency room visits for respiratory illnesses in Montreal, Quebec. Am J Crit Care Med 1997, 155(2):568-576.

25. Lipsett M, Hurley S y Ostro B. Air pollution and emergency room visits for asthma in Santa Clara County, California. Environ Health Perspect 1997, 105(2):216-222.

26. Alberdi JC y Díaz J. Modelización de la mortalidad diaria en la Comunidad Autónoma de Madrid (1986-1991). Gac Sanit 1997, 11(1):9-15.

27. Burnett RT, Cakmak S, Brook JR y Krewski D. The role of particulate size and chemistry in the association between summertime ambient air pollution and hospitalization for cardiorespiratory diseases. Environ Health Perspect 1997, 105(6): 614-620.

28. Ballester F, Corella D, Pérez-Hoyos S, Saez M y Hervás A. Mortality as a function of temperature. A study in Valencia, Spain, 1991-1993. Int J Epidemiol 1997, 26(3)551-561.

29. Besag J. Spatial interaction and the statistical analysis of lattice systems. J Royal Stat Soc B 1974, 36:192-236.

30. Diggle PJ, Liang KY y Zeger SL. Analysis of Longitudinal Data, Oxford: University Press; 1994. 